\title{
Quantum Mechanics of Gravitational Collapse
}

\author{
P. Hajicek \\ Institute for Theoretical Physics, University of Berne, Sidlerstrasse 5, \\ CH-3012 Berne, Switzerland
}

Received February 6, 1992

\begin{abstract}
A toy model of gravitational collapse in General Relativity is studied. It consists of a spherically symmetric thin shell of dust with a fixed rest mass. The configuration space is the half-axis and the Hamiltonian splits into a differential operator of infinite order ("free" Hamiltonian) and a "Coulomb" potential. Harmonic analysis on the half-axis is used to define the free Hamiltonian. For rest masses comparable to, or lower than one Planck mass, the Kato-Rellich theorem is applicable and one self-adjoint extension of the full Hamiltonian is found. A boundary condition for the wave function results whose effect is to keep the shell away from the singularity. This will lead to superposition of states containing both black and white holes.
\end{abstract}

\section{Introduction and Summary}

The classical theory of gravity, General Relativity, suffers from the problem of singularities. Gravitational collapse cannot be halted and it leads generically to infinite densities and curvatures. This divergence contradicts the basic postulates of the theory (e.g., locally Minkowskian spacetime, see e.g. [7]). A possible solution of this problem is often sought in quantum theory. However, attempts to construct quantum gravity have not been successful as yet.

In the present paper, we try to circumvent the construction of quantum gravity by working with a more tractable model, which still suffers in its classical form from the same disease. The model we choose is a spherically symmetric thin shell of dust with a fixed rest mass $M$ and its gravitational field as given by Einstein's equations. Thin shells have become quite popular as models for various phenomena in recent years (e.g. Refs. [1, 3, 15, and 19]).

In principle, there are at least two objections against the use of such minisuperspace models. First, suppressing most of the degrees of freedom can destroy some property of the original system which is relevant for the problem under study (cf. [12]). Thus, in our case, the result that there is no thermodynamics may be due to the freezing of all but one degree of freedom (see later). Second, canonical reduction of the degrees of freedom to just the physical ones needs a gauge fixing which, for 
parametrized systems like ours, includes a choice of time. However, it is wellknown that quantum theories based on different choices of time will in general not be unitarily equivalent ("multiple choice problem," see [11]). The criticism [4] of the choice of proper time along the shell world sheet seems to touch this problem. We will take an optimistic standpoint in this paper and assume that our minisuperspace model gives, at least qualitatively, valid hints of what happens in gravitational collapse.

The plan and the main results of the paper are as follows. In Sects. 2 and 3, we study the classical Hamiltonian and the corresponding dynamics of the shell and spacetime. The Hamiltonian is not bounded from below; the solutions with positive energy are asymptotically flat spacetimes with topology $\mathbb{R}^{3} \times \mathbb{R}$; the negative energies correspond to the spacetime topology $S^{3} \times \mathbb{R}$. Thus, two different space topologies are possible for this system. It is interesting that these two different topologies do not live in separated components of the configuration space $\mathscr{M}$ of the system: $\mathscr{M}=\mathbb{R}_{+},\left(\mathbb{R}_{+}=(0, \infty)\right)$, which is connected. The second important property is that the classical dynamics of the shell is incomplete: the shell collapses to the singularity in a finite proper time, or its world sheet cannot be extended to an arbitrary negative value of the proper time, as one again encounters a singularity. Moreover, during the collapse of the shell, a future event horizon always forms. Thus, there are singularities and black holes in this model as in General Relativity.

Any attempt to make classical dynamics complete by some condition at the singularity meets with great difficulties. For example, it is impossible to impose some "bounce from the wall" condition because of the causal structure of the spacetime. The shell ends up at the so-called future singularity of the Kruskal spacetime, and there is no (causal) way to extend the motion, because there are no points in the spacetime which lie to the future of the singularity. Thus, one has to extend the spacetime through the singularity. However, such an extension is a very uncertain business (see e.g. [17]): different spacetimes can be attached to the Kruskal one along its future singularity, a detailed way of how a particular one is to be attached is not well-defined, and how the shell world sheet is to proceed in the new spacetime is not determined. Physically, the shell undergoes an infinite squashing with infinite forces and the gravitational field experiences points with infinite curvature.

In Sect. 4, we define the Hilbert space and the basic operators for the system. Since the configuration space is $\mathbb{R}_{+}$, this definition is not straightforward. To deal with this problem we follow ref. [8]. Then, we choose a factor ordering in the Hamiltonian which seems to be the simplest one. We are confronted with an unusual situation of an operator which is of infinite order in derivatives. In Sect. 5, we describe the harmonic analysis on $\mathbb{R}_{+}$and construct by this tool a oneparameter family of self-adjoint extensions of the free Hamiltonian. The most important properties of the corresponding domains are: the wave functions must be $C_{0}^{\infty}([0, \infty))$, and must satisfy a rather strict boundary condition at the singularity:

$$
\psi^{(2 n)}(0) \sin \alpha+\psi^{(2 n+1)}(0) \cos \alpha=0 \quad \forall n=0,1, \ldots
$$

where $\alpha \in(-\pi, 0]$ is the parameter distinguishing the different extensions. The appearance of all derivatives is due to the infinite order of the operator.

In Sect. 6, we use the Kato-Rellich theorem (see e.g. [16]) to find a self-adjoint extension of the full Hamiltonian. This works only a) if the rest mass of the shell is 
comparable to, or lower than, about one Planck mass, and b) for one value of $\alpha$, namely $\alpha=-\frac{1}{2} \pi$. Then, the domain of the constructed full Hamiltonian coincides with the domain of the free one. We have no proof that this is the only possible extension. In any case, this particular extension leads to a particular boundary condition at the singularity, namely that $\psi^{(2 n)}(0)=0$ for all $n=0,1, \ldots$. This implies immediately that the probability $|\psi(0)|^{2}$ to find the shell at the singularity is zero. We also find the probability current $J(x)$, which is conserved as a consequence of the Schroedinger equation, and show that the above boundary condition leads to vanishing of this current at the singularity (this holds for all $\alpha$ ).

It is instructive to compare the boundary condition (1) in the quantum theory with the above described attempts to make the classical theory complete. (For a rigorous discussion of incompleteness of dynamics, classical and quantum, see [16], p. 146). First, the problem to make a quantum theory complete seems to be mathematically better defined than the corresponding classical one. One has to find a self-adjoint extension of the Hamiltonian; the question of how many self-adjoint extensions a given symmetric operator can possess, and what these extensions are has been, at least in principle, completely answered by von Neumann's theory of deficiency indices. In our particular case, we have so far only one extension, and it seems plausible that there could be at most a one-parameter family of them.

Second, usually (and it is so in our case) the domain of the extended operator contains only functions which satisfy certain conditions at the relevant boundaries. Now, the boundary condition (1) has no sensible analogy in the classical theory, because of the causal structure of the classical solutions: the quantum theory seems to disregard this structure. There is no paradox, however. The quantum system need not obey a causal structure of any fixed classical spacetime or even of a class of such spacetimes. The reason is that the full quantum dynamics of the system "shell \& gravitational field" is not a dynamics within a particular spacetime. The wave function contains information about the state of the gravitational field as well as that of the shell. The boundary condition at the singularity can lead to such a broad smearing of the wave packet, that a state of an infalling shell in a shrinking three-space (three-geometry is a point in the configuration space of gravity) is superposed with a state of an outgoing shell in an expanding three-space. That is, a state of a shell and a three-space falling into the future singularity is combined with that of the shell and three-space just leaving the past singularity. For shells that reach infinity, these two states cannot coexist within one classical spacetime: either the shell collapses and there is only a future singularity, or it expands and there is only a past one. This leads to a rather surprising conclusion: quantum evolution of a low-mass shell that starts at a given $\mathscr{I}$ can in principle be always finished at the same $\mathscr{I}$-no contradiction seems to result. However, more work will be necessary to understand this point.

One can also compare our boundary condition with those imposed in quantum cosmology: Linde's [14] and Vilenkin's conditions [18] and the Hawking-Hartle no-boundary proposal [6]. There are two differences. First, the causal structure of classical solutions describing processes involving black holes is very different from that of a cosmological model. One can be mislead by the presence of event horizon to believe that any condition at the singularity is superfluous: if the shell crosses the horizon, its fate is sealed, etc. However, black holes are leaky in the quantum theory, because they are smeared with white holes. Second, each of the conditions in cosmology is one of the main assumptions of the theory. Condition (1), however, is derived from another assumption, namely "The Principle of Unitarity' (for 
arguments in favor of unitarity in quantum gravity, see [9]). Thus, it is an "output" rather than an "input."

It seems quite plausible that there will be scattering states in the quantum mechanics we have constructed: a wave packet coming in from infinity, bouncing at the singularity and going back to infinity. What will be an interpretation of such a process? This will depend on the form of the wave function in a neighbourhood of the singularity. In particular, the relation

$$
\gamma=\langle\rho\rangle_{\min } /\left(2 l^{2}\langle H\rangle\right)
$$

between the minimal and the Schwarzschild radius of the shell seems to be a relevant parameter. For example, $\gamma$ could depend on the energy $\langle H\rangle$ in such a way that a threshold $E_{b}$ for the formation of black holes $(\gamma<1)$ would result (cf. [5]). Then, for the energies higher than $E_{b}$, the scattering state would describe some extreme form of the Hawking effect. However, as the evolution is unitary, no loss of information seems to result and no thermodynamics seems to be applicable. In any case, more work on this simple model is necessary before it can be completely understood.

\section{Self-Gravitating Spherically Symmetric Dust Shells}

In this section, we briefly introduce the classical model following closely ref. [1]. The classical paper on the dynamics of thin shells is ref. [10].

The world sheet $\Sigma$ of a thin shell is a common three-dimensional boundary of two spacetimes. Let us call these "out"- and "in"-spacetimes, $\mathscr{M}_{+}$and $\mathscr{M}_{-} . \mathscr{M}_{+}$and

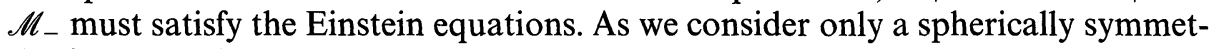
ric situation, they must be Schwarzschild spacetimes for two different mass parameters $m_{+}$and $m_{-}$. We assume that the shell is the only source of gravity; then, $\mathscr{M}_{-}$ is a part of Minkowski spacetime which does not contain the null infinity. Consequently, $m_{-}=0$, and $m=m_{+}$is the total mass of the system. On $\Sigma$, we can introduce the coordinates $\tau$ and $\rho$ such that the induced metric on $\Sigma$ takes the form:

$$
d s^{2}=-d \tau^{2}+\rho^{2}(\tau)\left(d \vartheta^{2}+\sin ^{2} \vartheta d \varphi^{2}\right) .
$$

Here, $\tau$ is the proper time along the world sheet and $\rho(\tau)$ is the radius of the shell at the time $\tau$. Finally, we suppose for the sake of simplicity that the shell consists of dust. Then, its 3-energy-momentum tensor takes the form

$$
S_{k l}=\frac{M}{4 \pi \rho^{2}} \delta_{k}^{0} \delta_{l}^{0}
$$

where $M$ is the total rest mass of the shell. Shells with different rest masses are different dynamical systems. $M$ is "constant" over the whole of the phase space, (it is not a dynamical variable), whereas $m$ is constant only along the dynamical trajectories (it is a dynamical variable).

The Einstein equations imply the following master equation for the motion of the shell:

$$
\sqrt{1+\dot{\rho}^{2}}-\sigma \sqrt{1+\dot{\rho}^{2}-2 l^{2} m \rho^{-2}}=l^{2} m \rho^{-1}
$$


Here, $\sigma$ is' a' sign giving information about what part of the Kruskal manifold with mass $m$ is taken as $\mathscr{M}_{+}: \sigma=+1$, if this part does not contain the two-surface where the two horizons cross each other, and $\sigma=-1$ otherwise. $l$ is the Planck length; the units are chosen such that $\hbar=c=1$.

From Eq. (2), one obtains the energy by solving it for $m$ :

$$
E(\rho, \dot{\rho})=M \sqrt{1+\dot{\rho}^{2}}-\beta \rho^{-1},
$$

where

$$
\beta=\frac{1}{2} l^{2} M^{2}
$$

The equation of motion for the shell is obtained by differentiating (3) and excluding the invalid root $\dot{\rho}=0$ :

$$
\frac{M}{\sqrt{1+\dot{\rho}^{2}}} \ddot{\rho}+\frac{\beta}{\rho^{2}}=0 .
$$

The classical Hamiltonian $h(\rho, \pi)$ which reproduces the equation of motion and the value (3) of the total energy is uniquely determined to be

$$
h(\rho, \pi)=M \cosh (\pi / M)-\beta / \rho,
$$

where $\pi$ is the momentum conjugate to $\rho$,

$$
\dot{\rho}=\sinh (\pi / M) \text {. }
$$

Let us just mention that a charged dust shell will have a Hamiltonian of the same form with

$$
\beta=\frac{1}{2}\left(l^{2} M^{2}-Q^{2}\right),
$$

where $Q$ is the electric charge of the shell (cf. [1]). Thus, many results obtained in the present paper apply as well to charged shells (but can have a different physical interpretation). The problem will be studied in a later paper.

We finish this section by introducing dimensionless quantities which will simplify further work. The coordinate $x$, the conjugate momentum $p$, the time $t$ and the Hamiltonian $H$ are defined as follows:

$$
\begin{gathered}
x=M \rho, \quad p=\pi / M, \\
t=M \tau, \quad H=h / M .
\end{gathered}
$$

Equations (4) and (5) become

$$
\begin{aligned}
H(x, p) & =\cosh p-\beta / x, \\
\dot{x} & =\sinh p .
\end{aligned}
$$

\section{Properties of the Classical Dynamics}

In this section, we are going to discuss some important features of the classical Hamiltonian (6). 
A) The dynamics is incomplete. We can easily show that each solution of Eq. (3) runs off to $\rho=0$ in a finite time, either forwards, backwards, or both. Indeed, we can write (3) in the form

$$
\dot{\rho}^{2}+V_{\text {eff }}(\rho)=0
$$

where

$$
V_{\text {eff }}(\rho)=1-\left(\frac{m \rho+\beta}{M \rho}\right)^{2} .
$$

$V_{\text {eff }}(\rho)$ is an increasing function of $\rho$ in the whole interval $[0, \infty)$, and

$$
V_{\text {eff }}(\infty)=1-m^{2} M^{-2} \text {. }
$$

Hence, there are only three types of solutions.

1) $m \geqq M, \dot{\rho}>0$. The world sheet of the shell starts at $\rho=0$ at a finite time $\tau_{-}$, and approaches $\rho=\infty$ with $\tau=+\infty$ and $\dot{\rho}=\sqrt{-V_{\text {eff }}(\infty)}$.

2) $m \geqq M, \dot{\rho}>0$. This motion is the time inversion of the first one.

3) $m<M$. The world sheet starts at $\rho=0$ at a time $\tau_{-}$and $\dot{\rho}=\infty$, reaches a turning point with

$$
\rho_{0}=\beta /(M-m)
$$

and falls back to $\rho=0$ at a time $\tau_{+}$and $\dot{\rho}=-\infty$.

These properties of the shell dynamics reflects the main problem of classical General Relativity: the gravitational collapse to the singularity. The part of the classical solution which describes the spacetime also becomes singular. It is just the Kruskal manifold with the value $m$ of the mass parameter.

B) $H$ is not bounded from below. We observe that the Hamiltonian (6) is not bounded from below on the phase space $\Gamma=\mathbb{R}_{+} \times \mathbb{R}, x \in \mathbb{R}_{+}, p \in \mathbb{R}$. For a relativistic theory, the Hamiltonian not only is to be bounded from below, but it is to be non-negative. For classical General Relativity, there is a proof of the positivity of the total energy [20]. It looks like a paradox, therefore, that our Hamiltonian which is a Hamiltonian of a general relativistic system - can be negative. The solution of the paradox is that our classical solutions with negative energy cannot be asymptotically flat (total energy is defined at the infinity of an asymptotically flat spacetime). Thus, the part of the Schwarzschild spacetime which plays the role of $\mathscr{M}_{+}$must not contain the infinity in these cases. For $m=0, \mathscr{M}_{+}$is simply the same part of the Minkowski spacetime as $\mathscr{M}_{-}$. If these manifolds are glued together along their isometrical boundaries, a spacetime with the topology $S^{3} \times \mathbb{R}$ will result. Similarly, for $m<0, \mathscr{M}_{+}$is that part of the Schwarzschild solution with negative mass, which contain the (timelike) central singularity. Again (if we add these singular points to the spacetime, for each value of the time coordinate one), we obtain the topology $S^{3} \times \mathbb{R}$.

This is a situation which is not quite common from at least two points of view. First, the "topology of the classical solution" for our system seems to allow two different values, but the phase space of the system is connected. Different topologies can exist in neighbouring points of the phase space! Second, a very important part of the physics of our system is clearly not determined by the structure of its Hamiltonian only. Knowing the expression (6), we can calculate the function $x(t)$. 
However, from this function or just from the expression (6), we cannot infer that there is a spacetime and what is its geometry. Neither does the expression (6) alone tell us anything about how the classical system avoids spending arbitrarily large energy. One can try to explain this situation as follows. By canonically reducing the system, one has to solve the constraints for the dependent variables. This is the geometry of the space in the present case. Then, one obtains this geometry (and so the topology) as some function of the "true dynamical variables" $x$ and $p$. Thus, the full classical solution - that is a motion of the shell in a spacetime - can be reconstructed only after this function is given together with the Hamiltonian.

\section{Quantization: The First Steps}

The problem of quantization of a system with a configuration space $\mathbb{R}_{+}$is not trivial. We will use the method described in ref. [8], p. 1162. Very briefly, the Hilbert space is $\mathscr{L}^{2}\left(\mathbb{R}_{+}, x^{-1} d x\right)$, and the basic observables are the coordinate $x$ and the phase space function $\Pi=x p$. These can be represented by the operators

$$
\begin{aligned}
x \psi(x) & =x \psi(x), \\
\Pi \psi(x) & =-i x \frac{d}{d x} \psi(x),
\end{aligned}
$$

which can be made self-adjoint. The next problem is to define $\cosh p$. Let us denote the corresponding operator by $H^{0}$ (the Hamiltonian of the "free theory"). We have

$$
\cosh p=\sum_{n=0}^{\infty} \frac{1}{(2 n) !} p^{2 n}
$$

Thus, $\cosh p$ is a function of $p^{2} \cdot p^{2}$ is expressed by means of $x$ and $\Pi$ as follows:

$$
p^{2}=\left(x^{-1} \Pi\right)^{2}
$$

and we choose the simplest factor ordering making the operator symmetric with respect to the measure $x^{-1} d x$ :

$$
-x^{1 / 2} x^{-1} \Pi x^{-1} \Pi x^{-1 / 2}=-x^{-1 / 2} \Delta x^{-1 / 2}
$$

where

$$
\Delta=(d / d x)^{2} .
$$

The following definition seems, therefore, natural

$$
H^{0}=x^{1 / 2}\left(\sum_{n=0}^{\infty} \frac{(-1)^{n}}{(2 n) !} \Delta^{n}\right) x^{-1 / 2}
$$

Many calculations will be simplified if we transform to $\mathscr{L}^{2}\left(\mathbb{R}_{+}, d x\right)$ using the unitary map

$$
U: \mathscr{L}^{2}\left(\mathbb{R}_{+}, x^{-1} d x\right) \rightarrow \mathscr{L}^{2}\left(\mathbb{R}_{+}, d x\right)
$$

defined by

$$
\psi(x) \rightarrow x^{-1 / 2} \psi(x)
$$


The operator (8) goes over into $-\Delta$ and (9) into

$$
H^{0}=\sum_{n=0}^{\infty} \frac{(-1)^{n}}{(2 n) !} \Delta^{n} .
$$

Thus, the study of the operator (9) in $\mathscr{L}^{2}\left(\mathbb{R}_{+}, x^{-1} d x\right)$ is equivalent to that of the operator $(10)$ in $\mathscr{L}^{2}\left(\mathbb{R}_{+}, d x\right)$. We can utilise the well-known self-adjoint extensions of $\Delta$ on $\mathscr{L}^{2}\left(\mathbb{R}_{+}, d x\right)$ and the fact that $H^{0}$ is a function of it.

\section{Harmonic Analysis on $\mathbb{R}_{+}$}

Harmonic analysis - that is, roughly speaking, the expansion in the generalized eigenfunctions of a Laplacian - will be a key to the properties of the Hamiltonian. We start this section by collecting relevant points about the Laplacian on $\mathbb{R}_{+}$and then pass to investigation of the free Hamiltonian.

The self-adjoint extensions $\Delta_{\alpha}$ of the Laplacian on $\mathbb{R}_{+}$can be parametrized by the parameter $\alpha \in(-\pi, 0]$ (see, e.g. [16], p. 144). The functions from the corresponding domains satisfy the following boundary condition at $x=0$ :

$$
\psi(0) \sin \alpha+\psi^{\prime}(0) \cos \alpha=0 \text {. }
$$

The complete orthonormal ( $\delta$-function-orthonormal) set of generalized eigenfunctions of $\Delta_{\alpha}$ have the form

$$
\varphi_{\alpha}(p, x)=a_{\alpha}(p) e^{-i p x}+b_{\alpha}(p) e^{i p x} .
$$

Equation (11) implies that

$$
\left[a_{\alpha}(p)+b_{\alpha}(p)\right] \sin \alpha+\left[-i p a_{\alpha}(p)+i p b_{\alpha}(p)\right] \cos \alpha=0
$$

for all p. From Eq. (13) and the normalization condition, we obtain (up to a phase factor):

$$
\begin{aligned}
& a_{\alpha}(p)=(2 \pi)^{-1 / 2} \frac{p \cos \alpha-i \sin \alpha}{\sqrt{p^{2} \cos ^{2} \alpha+\sin ^{2} \alpha}}, \\
& b_{\alpha}(p)=(2 \pi)^{-1 / 2} \frac{p \cos \alpha+i \sin \alpha}{\sqrt{p^{2} \cos ^{2} \alpha+\sin ^{2} \alpha}},
\end{aligned}
$$

or

$$
\varphi_{\alpha}(p, x)=(2 / \pi)^{1 / 2} \frac{p \cos \alpha \cos p x-\sin \alpha \sin p x}{\sqrt{p^{2} \cos ^{2} \alpha+\sin ^{2} \alpha}}
$$

In particular,

$$
\begin{aligned}
\varphi_{-\pi / 2}(p, x) & =(2 / \pi)^{1 / 2} \sin p x \\
\varphi_{0}(p, x) & =(2 / \pi)^{1 / 2} \cos p x .
\end{aligned}
$$

The corresponding generalization of the Fourier transformation is defined by

$$
\psi(x)=\int_{0}^{\infty} d p \tilde{\psi}(p) \varphi_{\alpha}(p, x)
$$


and

$$
\tilde{\psi}(p)=\int_{0}^{\infty} d x \psi(x) \varphi_{\alpha}^{*}(p, x)
$$

We have, for any $\alpha$, that

$$
\int_{0}^{\infty} d x|\psi(x)|^{2}=\int_{0}^{\infty} d p|\tilde{\psi}(p)|^{2} .
$$

We consider Eqs. (15) and (16) as defining a unitary transformation, $F_{\alpha}$, and its inverse, $F_{\alpha}^{-1}$,

$$
F_{\alpha}: \mathscr{L}^{2}\left(\mathbb{R}_{+}, d p\right) \rightarrow \mathscr{L}^{2}\left(\mathbb{R}_{+}, d x\right) .
$$

The transform of $H^{0}$ is simply

$$
\left(F_{\alpha}^{-1} H^{0} F_{\alpha} \tilde{\psi}\right)(p)=\cosh p \tilde{\psi}(p) .
$$

The natural domain for this operator in $\mathscr{L}^{2}\left(\mathbb{R}_{+}, d p\right)$ is $C_{0}^{\infty}\left(\mathbb{R}_{+}\right): C_{0}^{\infty}\left(\mathbb{R}_{+}\right)$is dense in $\mathscr{L}^{2}\left(\mathbb{R}_{+}, d p\right), \cosh p \tilde{\psi}(p)$ is square integrable for any $\widetilde{\psi}(p) \in C_{0}^{\infty}\left(\mathbb{R}_{+}\right)$and $\cosh p$ is symmetric on $C_{0}^{\infty}\left(\mathbb{R}_{+}\right)$. Let us denote by $H_{\alpha}^{0}$ the symmetric operator $H^{0}$ with the domain $F_{\alpha}\left(C_{0}^{\infty}\left(\mathbb{R}_{+}\right)\right)$. The next two theorems describe important properties of the domains.

Theorem 1. Each $\psi(x) \in F_{\alpha}\left(C_{0}^{\infty}\left(\mathbb{R}_{+}\right)\right)$is an entire analytic function of $x$.

Proof. Equation (15) can be considered as an ordinary Fourier transform of some function on the whole real axis. Indeed, define the map,

$$
\tilde{\chi}_{\alpha}: \mathscr{L}^{2}\left(\mathbb{R}_{+}, d p\right) \rightarrow \mathscr{L}^{2}(\mathbb{R}, d p),
$$

by

$$
\begin{aligned}
\left(\tilde{\chi}_{\alpha} \tilde{\psi}\right)(p) & =2^{-1 / 2} \frac{p \cos \alpha-i \sin \alpha}{\sqrt{p^{2} \cos ^{2} \alpha+\sin ^{2} \alpha}} \tilde{\psi}(p) \text { for } p>0, \\
& =2^{-1 / 2} \frac{-p \cos \alpha+i \sin \alpha}{\sqrt{p^{2} \cos ^{2} \alpha+\sin ^{2} \alpha}} \tilde{\psi}(-p) \text { for } p<0 .
\end{aligned}
$$

First, $\tilde{\chi}_{\alpha}$ is clearly an isometry. Second, we easily see that $\tilde{\chi}_{\alpha}$ maps $C_{0}^{\infty}\left(\mathbb{R}_{+}\right)$on $C_{0}^{\infty}(\mathbb{R})$ as $\tilde{\psi}(p) \in C_{0}^{\infty}\left(\mathbb{R}_{+}\right)$must vanish in a whole interval $[0, \varepsilon), \varepsilon>0$. Finally,

$$
\psi(x)=\pi^{-1 / 2} \int_{-\infty}^{\infty} d p\left(\tilde{\chi}_{\alpha} \tilde{\psi}\right)(p) e^{-i p x}, \quad x>0 .
$$

Then, as $\left(\tilde{\chi}_{\alpha} \tilde{\psi}\right)(p) \in C_{0}^{\infty}(\mathbb{R})$, the Paley-Wiener theorem (see e.g. [16], p. 16) implies the claim, QED.

Theorem 2. Each $\psi(x) \in F_{\alpha}\left(C_{0}^{\infty}\left(\mathbb{R}_{+}\right)\right)$satisfies Eq. (1),

$$
\psi^{(2 n)}(0) \sin \alpha+\psi^{(2 n+1)}(0) \cos \alpha=0
$$

for all non-negative integers $n$. 
Proof. Let $\tilde{\psi}(p) \in C_{0}^{\infty}\left(\mathbb{R}_{+}\right)$; then

$$
\psi^{(k)}(0)=\int_{0}^{\infty} d p \tilde{\psi}(p)\left[(-i p)^{k} a_{\alpha}(p)+(i p)^{k} b_{\alpha}(p)\right]
$$

for each integer $k$. Thus,

$$
\begin{aligned}
& \psi^{(2 n)}(0) \sin \alpha+\psi^{(2 n+1)}(0) \cos \alpha \\
& =\int_{0}^{\infty} d p \tilde{\psi}(p)\left[(-i p)^{2 n} a_{\alpha}(p) \sin \alpha+(i p)^{2 n} b_{\alpha}(p) \sin \alpha\right. \\
& \left.\quad+(-i p)^{2 n+1} a_{\alpha}(p) \cos \alpha+(i p)^{2 n+1} b_{\alpha}(p) \cos \alpha\right] \\
& =\int_{0}^{\infty} d p \tilde{\psi}(p)(-1)^{n} p^{2 n}\left[\left(a_{\alpha}(p)+b_{\alpha}(p)\right) \sin \alpha\right. \\
& \left.\quad+\left(-i p a_{\alpha}(p)+i p b_{\alpha}(p)\right) \cos \alpha\right] .
\end{aligned}
$$

The claim follows immediately from Eq. (13).

A simple corollary of Theorems 1 and 2 is that

$$
F_{\alpha}\left(C_{0}^{\infty}\left(\mathbb{R}_{+}\right)\right) \cap F_{\alpha^{\prime}}\left(C_{0}^{\infty}\left(\mathbb{R}_{+}\right)\right)=\{0\}
$$

for $\alpha \neq \alpha^{\prime}$. Indeed, an analytic function is uniquely determined by its Taylor expansion at the origin, and any pair of equations of the form (1) with $\alpha \neq \alpha$ have only the zero solution in common. Another consequence is that

$$
H_{\alpha}^{0} \psi(x)=\frac{1}{2} \psi(x+i)+\frac{1}{2} \psi(x-i)
$$

for any $\psi \in F_{\alpha}\left(C_{0}^{\infty}\left(\mathbb{R}_{+}\right)\right)$.

The next task is to find self-adjoint extensions of the symmetric operators $H_{\alpha}^{0}$. We can use von Neumann's theory of deficiency indices (see, e.g. [16], p. 135) to obtain the following theorem.

Theorem 3. The operator $H_{\alpha}^{0}$ with the domain $F_{\alpha}\left(C_{0}^{\infty}\left(\mathbb{R}_{+}\right)\right)$is essentially selfadjoint for each $\alpha$.

Proof. The deficiency subspaces $\mathscr{K}_{ \pm}$of $H_{\alpha}^{0}$ are defined by

$$
\mathscr{K}_{ \pm}=\left[\operatorname{Ran}\left(H_{\alpha}^{0} \pm i\right)\right]^{\perp} .
$$

Let us consider the right-hand side in $\mathscr{L}^{2}\left(\mathbb{R}_{+}, d p\right)$. The operator $H_{\alpha}^{0}$ is represented by multiplication by $\cosh p$ there. It is obvious that

$$
(\cosh p \pm i) \tilde{\psi}(p) \in C_{0}^{\infty}\left(\mathbb{R}_{+}\right)
$$

if $\tilde{\psi}(p) \in C_{0}^{\infty}\left(\mathbb{R}_{+}\right)$. Moreover, cosh $p \pm i$ is bounded below from zero on the real axis:

$$
|\cosh p \pm i|^{2} \geqq 2
$$

It follows that

$$
(\cosh p \pm i) C_{0}^{\infty}\left(\mathbb{R}_{+}\right)=C_{0}^{\infty}\left(\mathbb{R}_{+}\right)
$$


and

$$
\operatorname{Ran}(\cosh p \pm i)=C_{0}^{\infty}\left(\mathbb{R}_{+}\right)
$$

However, $C_{0}^{\infty}\left(\mathbb{R}_{+}\right)$is dense in $\mathscr{L}^{2}\left(\mathbb{R}_{+}, d p\right)$ and so only the zero vector can be orthogonal to it. QED.

The self-adjoint extension of an essentially self-adjoint operator is obtained by closure. Will the boundary condition (1) survive the operation of closure? The corresponding domain is the completion of the $\operatorname{Dom}\left(H_{\alpha}^{0}\right)$ with respect to the "scalar product" (see e.g. [16], p. 138)

$$
(\varphi, \psi)_{\alpha}=(\varphi, \psi)+\left(H_{\alpha}^{0} \varphi, H_{\alpha}^{0} \psi\right) \text {. }
$$

Thus, the Fourier component of each element of $\operatorname{Dom}\left(\bar{H}_{\alpha}^{0}\right)$ must be integrable with $\cosh ^{2} p$ (as usual, $\bar{A}$ denotes the closure of the operator $A$ ):

$$
\int_{0}^{\infty} d p|\tilde{\psi}(p)|^{2} \cosh ^{2} p<\infty
$$

Then,

$$
\int_{0}^{\infty} d p|\tilde{\psi}(p)|^{2} p^{n}<\infty
$$

for all $n \geqq 0$. Thus, we can differentiate behind the sign of integral similarly as in the proof of the Theorem 2 to show the following:

Theorem 4. Let $\psi(x) \in \operatorname{Dom}\left(\bar{H}_{\alpha}^{0}\right)$. Then,

$$
\psi(x) \in C^{\infty}[0, \infty),
$$

and $\psi(x)$ satisfies the boundary condition (1).

It follows that $\operatorname{Dom}\left(\bar{H}_{\alpha}^{0}\right)$ and $\operatorname{Dom}\left(\bar{H}_{\alpha^{\prime}}^{0}\right)$ can have non-trivial common elements, but all derivatives of these functions must vanish at the origin. Moreover, Eq. (17) holds on the core of $\bar{H}_{\alpha}^{0}$ for any $\alpha$. In ref. [2], all solutions of the "eigenvalue equation"

$$
\frac{1}{2} \psi(x+i)+\frac{1}{2} \psi(x-i)=\varepsilon \psi(x)
$$

have been found. In particular, $\psi(x)=e^{-\lambda x}$ is a square integrable solution for any $\lambda>0$ with $\varepsilon=\cos \lambda$. There is an infinite "degeneration" for a fixed value of $\varepsilon$ :

$$
\lambda=\arccos \varepsilon+2 n \pi, \quad n \in \mathbb{Z}_{+} .
$$

However, these functions do not satisfy Eq. (1), and so they are not eigenfunctions of $\bar{H}_{\alpha}^{0}$ for any $\alpha$. Of course, the generalized eigenfunctions of $\bar{H}_{\alpha}^{0}$ are given by Eq. (12) or (13); the free Hamiltonian has a purely continuous spectrum.

Thus, we have obtained quite a complete theory of the free Hamiltonian. It will be applied in the next section. 


\section{Construction of a Unitary Quantum Theory}

The harmonic analysis on $\mathbb{R}_{+}$was a useful tool for the construction of the free theory. For the interacting theory, we need a new idea. We will find it in the Kato-Rellich theorem (see e.g. [16], p. 162). The application of this theorem to the full Hamiltonian $H$ gives the following:

Theorem 5. The operator

$$
H=\bar{H}_{-\pi / 2}^{0}-\beta x^{-1}
$$

with the domain $\operatorname{Dom}\left(\bar{H}_{-\pi / 2}^{0}\right)$ is self-adjoint, if

$$
\beta<(\pi / 2)^{1 / 2} \kappa
$$

where

$$
\kappa=\min _{p \in \mathbb{R}_{+}} \frac{\cosh p}{p} .
$$

(As $\kappa \approx 1.51$, the mass of the shell has to be smaller than about two Planck masses.) Proof. The Kato-Rellich theorem has two conditions, which read in our case:

and

$$
\operatorname{Dom}\left(\bar{H}_{\alpha}^{0}\right) \subset \operatorname{Dom}\left(-\beta x^{-1}\right),
$$

$$
\left\|-\beta x^{-1} \psi\right\|^{2} \leqq A^{2}\left\|\bar{H}_{\alpha}^{0} \psi\right\|^{2}+B^{2}\|\psi\|^{2}
$$

for all $\psi \in \operatorname{Dom}\left(H_{\alpha}^{0}\right) \cap \operatorname{Dom}\left(-\beta x^{-1}\right)$ with $A<1$ and $B \in \mathbb{R}$. The condition (20) can only be satisfied for $\alpha=-\pi / 2$, as Eq. (1) then implies that $\psi(0)=0$, and the Theorem 4 guarantees that $\psi^{\prime}(0)$ exists. For other $\alpha$ 's, $\operatorname{Dom}\left(\bar{H}_{\alpha}^{0}\right)$ contains functions with $\psi(0) \neq 0$, so that $\left\|-\beta x^{-1} \psi\right\|^{2}$ diverges.

The inequality (21) can be shown for any $\alpha$ if $\beta$ satisfies (19). Consider $\psi \in$ $\operatorname{Dom}\left(H_{\alpha}^{0}\right) \cap \operatorname{Dom}\left(-\beta x^{-1}\right)$. The condition (1) implies that this is equivalent to $\psi \in \operatorname{Dom}\left(H_{\alpha}^{0}\right)$ and $\psi(0)=0$. Then,

$$
\psi(x)=\int_{0}^{\infty} d p \tilde{\psi}(p) \varphi_{\alpha}(p, x),
$$

where $\tilde{\psi}(p) \in C_{0}^{\infty}\left(\mathbb{R}_{+}\right)$and satisfies the equation

$$
\int_{0}^{\infty} d p \tilde{\psi}(p) \varphi_{\alpha}(p, 0)=0 .
$$

Hence, we can write

$$
x^{-1} \psi(x)=\int_{0}^{\infty} d p \tilde{\psi}(p) \frac{\varphi_{\alpha}(p, x)-\varphi_{\alpha}(p, 0)}{x},
$$

Using Eq. (14), we find easily that

$$
\begin{aligned}
\frac{\varphi_{\alpha}(p, x)-\varphi_{\alpha}(p, 0)}{x}= & -(\pi / 2)^{-1 / 2} p\left[\frac{p \cos \alpha}{\sqrt{p^{2} \cos ^{2} \alpha+\sin ^{2} \alpha}} \frac{\sin (p x / 2)}{p x / 2} \sin (p x / 2)\right. \\
& \left.+\frac{\sin \alpha}{\sqrt{p^{2} \cos ^{2} \alpha+\sin ^{2} \alpha}} \frac{\sin p x}{p x}\right] .
\end{aligned}
$$


Substituting this into (22), we obtain

$$
\left|x^{-1} \psi(x)\right|^{2} \leqq(2 / \pi) \int_{0}^{\infty} d p|p \tilde{\psi}(p)|^{2} .
$$

The above definition of $\kappa$ yields that $|p| \leqq \kappa^{-1} \cosh p$, so

$$
\left|x^{-1} \psi(x)\right|^{2} \leqq\left(2 / \pi \kappa^{2}\right) \int_{0}^{\infty} d p|\cosh p \tilde{\psi}(p)|^{2} .
$$

Integrating this inequality from 0 to 1 , one finds

$$
\int_{0}^{1} d x\left|x^{-1} \psi(x)\right|^{2} \leqq\left(2 / \pi \kappa^{2}\right)\|\cosh p \tilde{\psi}(p)\|^{2} .
$$

The following inequality holds

$$
\int_{1}^{\infty} d x\left|x^{-1} \psi(x)\right|^{2} \leqq \int_{1}^{\infty} d x|\psi(x)|^{2} \leqq \int_{0}^{\infty} d x|\psi(x)|^{2} .
$$

The inequalities (23) and (24) imply the claim immediately. QED.

In this way, we have managed to construct at least one self-adjoint Hamiltonian $H$. The corresponding quantum mechanics will be unitary; the evolution will be given by the Schroedinger equation

$$
i \frac{\partial}{\partial \tau} \psi=H \psi
$$

The wave functions $\psi$ must be $C^{\infty}$ and must satisfy the condition

$$
\psi^{(2 n)}(0)=0, n=0,1, \ldots, 1 .
$$

It is, however, difficult to see, at the present moment, the significance of the fact that we could construct such a mechanics only for very light shells (very light indeed!). Either it exists for heavier shells as well, but one had to use different methods to find it, or there are no self-adjoint extension of $H$ for the heavy shells. The last case would be analogous to that of the Dirac electron in an external Coulomb field: if the central charge becomes $137 e$ or larger, then there is no self-adjoint extension (see, e.g. [13]). The physical interpretation is that electrons with low value of angular momenta fall on the center. In fact, it is quite plausible that a zero mode appears among the eigenfunctions of our Hamiltonian if the mass increases, and that this happens in a neighbourhood of the Planck mass.

We can get a nice interpretation of the boundary conditions (1), if we observe that there is a conserved probability current $J(x)$ for the Schroedinger equation (26). $J(x)$ is given as usual by

$$
J^{\prime}(x)=i\left(\psi^{*} H \psi-\psi H \psi^{*}\right),
$$

so that

$$
\left(\psi^{*} \psi\right)^{*}+J^{\prime}=0 \text {. }
$$

We can calculate $J$, if we write the right-hand side of (26) as follows:

$$
\sum_{n=0}^{\infty} \frac{(-1)^{n}}{(2 n) !} \psi^{(2 n)}(x)-\beta x^{-1} \psi(x),
$$


and the series converges (at least for the function from the core of the operator). Then, we obtain easily

$$
J=i \sum_{n=1}^{\infty} \frac{(-1)^{n}}{(2 n) !} \sum_{k=1}^{n}(-1)^{k-1}\left[\psi^{*(k-1)} \psi^{(2 n-k)}-\psi^{(k-1)} \psi^{*(2 n-k)}\right] .
$$

We show that $J(0)=0$ in consequence of the condition (1). Indeed, if $n=2 m$, the $k$-sum in (28) can be written as follows:

$$
\begin{aligned}
& \sum_{l=1}^{m}\left[\left(\psi^{*(2 l-2)} \psi^{(2 n-2 l+1)}-\psi^{*(2 l-1)} \psi^{(2 n-2 l)}\right.\right. \\
& \left.+\left(\psi^{(2 l-1)} \psi^{*(2 n-2 l)}-\psi^{(2 l-2)} \psi^{*(2 n-2 l+1)}\right)\right],
\end{aligned}
$$

whereas, if $n=2 m+1$, we obtain the same expression plus the following additional term:

$$
\left(\psi^{*(2 m)} \psi^{(2 m+1)}-\psi^{(2 m)} \psi^{*(2 m+1)}\right) .
$$

We can see immediately that all round brackets in the above expressions vanish if Eq. (1) holds, and $J=0$. Hence, the shell cannot cross the singularity.

In ref. [1], some square integrable solutions to the following equation:

$$
\frac{1}{2} \psi(x+i)+\frac{1}{2} \psi(x-i)-\beta x^{-1} \psi(x)=\varepsilon \psi(x)
$$

have been found. What is the significance of these? The left-hand side of Eq. (29) can be considered as $H \psi$, if $\psi$ is from the core of the operator. Thus, the solutions could be eigenvectors of $H$ and describe some bounded states. However, they do not satisfy the boundary condition (27). Moreover, one easily verifies that they are not orthogonal to each other. Hence, they are not eigenvectors of any self-adjoint operator whatsoever. In any case, there is no proof that our Hamiltonian has any bounded states.

Acknowledgement. Useful discussions with D. Giulini, A. Held, B.S. Kay, C. Kiefer, K.V. Kuchar, H. Leutwyler and E.A. Martinez are acknowledged. The work was supported in part by the Swiss Nationalfonds.

\section{References}

1. Berezin, V.A.: Quantum Black Holes and Hawking Radiation. Preprint, Bures-sur-Yvette, 1991

2. Berezin, V.A., Koziminov, N.G., Kuzmin, V.A., Tkachev, I.I.: Phys. Lett. B212, 415 (1988)

3. Farhi, E., Guth, A.H., Guven, J.: Nucl. Phys. B339, 417 (1990)

4. Guven, J.: Phys. Rev. D44, 3360 (1991)

5. Hajicek, P.: Phys. Rev. D30, 1168 (1984)

6. Hartle, J.B., Hawking, S.W.: Phys. Rev. D28, 2960 (1983)

7. Hawking, S.W., Ellis, G.F.R.: The Large Scale Structure of Space-Time. Cambridge: Cambridge University Press 1973

8. Isham, C.J.: In: Relativity, Groups and Topology II. DeWitt, B.S., Stora, R. (eds.). Amsterdam: North-Holland 1984

9. Jacobson, T.: In: Conceptual Problems of Quantum Gravity. Ashtekar, A., Stachel, J. (eds.). Boston: Birkhauser 1991 
10. Israel, W:: Nuovo Cimento 44B, 1 (1966)

11. Kuchar, K.V.: Time and Interpretation of Quantum Gravity. Preprint, University of Utah, SLC, 1991

12. Kuchar, K.V., Ryan, M.P.: In: Gravitational Collapse and Relativity. Sato, H., Nakamura, T. (eds.). Singapore: World Scientific 1986

13. Landau, L.D., Lifshitz. E.M.: Course of Theoretical Physics. Vol. 4, Part 1: Beresteckii, V.B., Lifshitz, E.M., Pitaevskii, L.P.: Relativistic Quantum Theory. Oxford: Pergamon Press, p. 110,1971

14. Linde, A.D.: Lett. Nuovo Cimento 39, 401 (1984)

15. Martinez, E.A., York, J.: Phys. Rev. D40, 2124 (1989)

16. Reed, M., Simon, B.: Methods of Modern Mathematical Physics. Vol. 2. Fourier Analysis, Self-Adjointness. New York: Academic Press 1975

17. Tipler, F.J., Clarke, C.J.S., Ellis, G.F.R.: In: General Relativity and Gravitation. One Hundred Years after the Birth of Albert Einstein. Held, A. (ed.). New York: Plenum Press 1980

18. Vilenkin, A.: Phys. Rev. D37, 888 (1988)

19. Visser, M.: Phys. Rev. D43, 402 (1991)

20. Witten, E.: Commun. Math. Phys. 80, 381 (1981)

Communicated by N. Yu. Reshetikhin 
